\title{
Comorbilidad en alcohólicos ingresados. Implicaciones clínicas y sociosanitarias.
}

\author{
Valbuena, A.; Largo, R.; Quintero-Gutierrez, J.; García-Resa, E.; Correas, J. \\ Servicio de Psiquiatría. Hospital Ramón y Cajal. Madrid. \\ Enviar correspondencia a: \\ A.Valbuena. C/Larra 15. 2D 28004. Madrid. Tf: 915320295
}

\section{RESUMEN:}

Del estudio retrospectivo de 188 ingresos con el diagnóstico de alcoholismo (DSM-IV) en el Servicio de Psiquiatría de un hospital general durante el trienio 1996-1998, se aprecia una importante comorbilidad tanto de enfermedades físicas como psiquiátricas. La comorbilidad y el peso asistencial varían según el tipo de alcoholismo. En una revisión pormenorizada de las historias clínicas y de acuerdo con los criterios CIE-10 (OMS) de consumo de alcohol los tres diagnósticos encontrados fueron: Intoxicación Etílica(E.I.), Consumo Perjudicial(C.P.) y Dependencia(D). Cada uno de los tres grupos presentan su propio perfil con diferencias sociodemográficas y en las enfermedades físicas y psiquiátricas asociadas. El grupo E.I. era el de menor edad, presentaba mejor arraigo sociofamiliar, tenía estancias muy cortas y no presentaba comorbilidad. El grupo C.P. tenía una edad media de 34 años, evidentes signos de desarraigo sociolaboral y una importante comorbilidad psiquiátrica y presentaba mayor peso asistencial. EL grupo D era el de mayor edad, presentaba comorbilidad física elevada, buena integración familiar pero mala laboral y el diagnóstico psiquiátrico mas frecuente era el trastorno afectivo.

La no inclusión del tipo de alcoholismo, según el DSM-IV, lleva a errores de apreciación e interpretación en muestras amplias de alcohólicos, errores que hemos encontrada con frecuencia en la revisión bibliográfica.

Palabras clave: alcoholismo. comorbilidad, estudio retrospectivo, DSM-IV, CIE-10

\section{ABSTRACT:}

A significant comorbility can be seen in a retrospective analysis of 188 admissions, diagnosed as alcoholism (DSM-IV), in the Psychiatric Unit of a General Hospital during the period from 1996-1998, in both physical and psychiatric illnesses. Comorbidity and admission loading varied according to the type of alcoholism. In a detailed review of the clinical histories, and in accordance with the (WHO) ICD-10 criteria on alcohol consumption, the three diagnoses found were: Drunkenness (DR), Harmful Use (HU) and Dependency (D). Each of the three groups presented a specific profile with sociodemographic differences, and in the associated physical and psychiatric illnesses. The DR Group was the youngest, presented more sociofamiliar integration, had very short admissions, and did not present comorbidity. The HU Group had an average age of 34 years, obvious signs of a lack of sociolaboral integration and a significant psychiatric comorbidity, and presented greater admission loading. The D Group was the oldest, presented high physical comorbidity, good family but bad occupational integration, and the most frequent psychiatric diagnosis was that of affective disorder.

Not including the type of alcoholism, according to the DSM-IV, leads to errors of evaluation and interpretation in large numbers of samples of alcoholics, errors that we frequently found in reviewing the literature.

Key words: alcoholism, comorbility, retrospective study, DSMIV, ICD-10.

\section{INTRODUCCIÓN.}

$\mathbf{L}$ a descripción clínica del alcoholismo lleva consigo su asociación con otros cuadros psiquiátricos. En un manual de reconocido prestigio como es el DSM, en su versión IV(1), se citan como trastornos mentales asociados los siguientes cuadros:

- Dependencia y abuso de otras sustancias( canna bis, cocaína, heroína, anfetaminas,
- sedantes, hipnóticos y ansiolíticos)

- Depresión, ansiedad e insomnio.

- Aumento del riesgo de accidentes, violencia y sui cidio.

- Trastorno antisocial de la personalidad, cuya aso ciación aumenta la probabilidad de actos criminal les.

- Trastorno del estado del ánimo. 
- Trastorno de ansiedad.

- Esquizofrenia.

- Problemas sociales, desarraigo laboral, ruptura familiar.

Estas alteraciones se consideran usualmente coincidentes con el cuadro de enfermedad alcohólica; se detectan tanto, si estudiamos su presencia en el momento determinado del estudio, como si hacemos un estudio longitudinal a lo largo de la vida. El trabajo de Arolt y Driessen (2) realizado en Alemania, en enfermos ingresados en un Hospital General, encuentra que aquellos pacientes en los que se podía hacer un diagnóstico de alcoholismo en el momento del estudio, presentaban una comorbilidad psiquiátrica del $44,4 \%$. Aquellos enfermos en los que se podía hacer un diagnóstico de alcoholismo a lo largo de su vida, previamente al ingreso, presentaban otro diagnóstico psiquiátrico en una proporción del $41 \%$. Este trabajo indica tambien que hay que distinguir entre dos tipos de patologías en relación con el alcoholismo y que vamos a citar aunque sean bien conocidas:

A - Aquellas enfermedades producidas directamente por el consumo de alcohol, que a su vez se diferencian en:

I- Patología somática secundaria al consumo del alcohol.

II- Patología psiquiátrica secundaria al consumo del alcohol( Delirium, Amnesia, Demencia, Alucinosis, etc.) que entraría en el apartado de trastorno orgánico secundario al consumo de alcohol.

B - Enfermedades psiquiátricas simultáneas a la enfermedad.

Siendo éste último grupo el objeto del presente estudio, al ser materia de interés en la bibliografía internacional $(3,4)$.

Otro aspecto consiste en la importancia sociosanitaria que plantea el consumo de alcohol en las sociedades occidentales. En un estudio previo (5), comprobábamos el peso asistencial del alcoholismo en el Hospital donde trabajamos. Se encontró que el diagnóstico de alcoholismo estaba asociado aproximadamente a un millar de ingresos al año, lo que representa una carga asistencial considerable, teniendo en cuenta que la patología somática asociada era de especial gravedad: enfermedades del aparato digestivo, enfermedades cardio-vasculares, traumatismos, accidentes de tráfico y diversos tumores $(6,7)$. El gasto producido en el ámbito hospitalario de toda España, se ha calculado en cerca de los cien mil millones de pesetas anuales (8). No es sorprendente que las autoridades sanitarias consideren el consumo patológico de alcohol como uno de los retos dentro de su competencia, como se menciona en el Plan Nacional Sobre Drogas. Un objetivo sanitario podría intentar graduar el consumo de alcohol hacia su reducción, de forma que supusiera una carga sanitaria menor. Como psiquiatras, que trabajamos integrados en la Red Sanitaria General, tendríamos la posibilidad de influir en este sentido a través de nuestro trabajo cotidiano. Por su indudable influencia en el coste económico-sanitario, el alcoholismo es y debe seguir siendo objeto de estudio e interés.

En los últimos diez años se ha prestado un interés creciente al problema de la comorbilidad en psiquiatría y en la enfermedad alcohólica en particular $(2,9,10$, $11,12)$. Aunque en el ámbito teórico se puede considerar que el alcoholismo es un fenómeno o enfermedad aislada, la práctica psiquiátrica nos indica la existencia de distintas conductas de consumo de alcohol, como formas de expresión de diversas enfermedades $(13,14)$. Recordamos que se reconoce clínicamente que otros trastornos psiquiátricos están habitualmente asociados al diagnóstico de alcoholismo.

Algunos cuadros psiquiátricos de reciente interés como el Trastorno por Estrés Postraumatico $(13,15)$, o los Ataques de Pánico, son las últimas líneas de estudio de la presencia de alcoholismo asociado. También lo son los diferentes grupos de población, como, mujeres $(16,17,18,19)$, jóvenes $(3,20,21,22)$, o ancianos (23). Todo ello refleja el interés general que plantean la patología psiquiátrica y los datos sociodemográficos asociados al consumo patológico de bebidas alcohólicas.

\section{MATERIAL Y METODO}

Se estudiaron de forma retrospectiva todos los diagnósticos relacionados con el consumo de sustancias alcohólicas en los enfermos ingresados en el Servicio de Psiquiatría del Hospital Ramón y Cajal, durante los años 1996, 1997 y 1998. Las historias clínicas fueron examinadas por los autores. El primer paso consistió en realizar un diagnóstico de la conducta alcohólica siguiendo las indicaciones de la OMS, en la Clasificación Internacional de las Enfermedades en su Décima Revisión sobre Trastornos Mentales y del Comportamiento. Para simplificar se ha utilizado como diagnóstico-base el tipo de consumo de las bebidas alcohólicas según los criterios de Intoxicación(I), Consumo Perjudicial(CP), ( que incluye Abuso según el DSM-IV), y Dependencia(D). Otras complicaciones debidas al alcoholismo forman parte del estudio.

Durante los tres años considerados para el estudio se registraron 232 ingresos en el Servicio de Psiquiatría, en los se reflejaba algún tipo de problema con el 
consumo de bebidas alcohólicas, de acuerdo con el listado anual realizado por el Servicio de Archivos del Hospital. Del examen de las historias clínicas se descartaron 44 ingresos por estar incompletas o mal codificadas, quedando 188 como objeto del estudio. Las causas por las que se desecharon los casos fueron:

-diagnóstico incorrecto.

-falta de datos ( pérdida de historia, insuficiente información en general).

-estancia insuficiente (enfermos trasladados a otros hospitales, con la consiguiente pérdida de información).

Los 188 protocolos de ingreso que constituyen el objeto del estudio fueron examinados de acuerdo con un protocolo en el que se recogían los siguientes datos.

\section{Datos generales:}

Diagnóstico, Sexo, Edad, Tipo de convivencia, Nivel de estudios, Situación laboral.

\section{2-Enfermedades somáticas:}

En donde se recogían exclusivamente aquellas enfermedades relacionadas con el consumo de alcohol.

\section{3-Enfermedades psiquiátricas:}

Divididas a su vez en

3-a) enfermedades psiquiátricas producidas por alcoholismo

3-b) Otras enfermedades psiquiátricas.

4-Variables asistenciales Ingresos previos y Estancia media.

El análisis de los resultados se planteó desde un punto meramente descriptivo de los ingresos producidos por el alcoholismo, agrupando los diagnósticos y observando la patología y otros datos asociados. En todo caso el número de observaciones en algunas tablas resultó inferior a cinco por lo que no es adecuado aplicar el contraste chi2.

\section{RESULTADOS.}

Del análisis de las 188 historias de enfermos ingresados en el servicio de Psiquiatría de un Hospital General, durante el trienio 1996-98, se han extraído los siguientes datos de acuerdo con el cuestionario desarrollado específicamente para este trabajo. Nos hemos encontrado con la dificultad del diagnóstico de
Abuso, según el DSM-IV, aunque como recomienda este manual, dicho diagnóstico es superponible al de Consumo Perjudicial de la CIE-10. Como podría ser evidente, algunos enfermos reunían criterios para los tres posibles diagnósticos. En estos casos siempre se utilizó el diagnóstico mas grave.

Los casos de Intoxicación(I) suponen 5 ingresos y aunque no permiten una comparación estadística por su escaso número, se han mantenido en las tablas con el fin de buscar posibles relaciones (Ver Tabla 1).

Tabla 1. Número de casos por diagnóstico. CIE-10

\begin{tabular}{||l|l||}
\hline Dependencia Alcohólica (D) & $79(42 \%)$ \\
\hline Consumo perjudicial (CP) & $104(55 \%)$ \\
\hline Intoxicación (I) & $5(3 \%)$ \\
\hline Total & $188(100 \%)$ \\
\hline
\end{tabular}

A continuación se agrupa a los enfermos en relación con los datos indicados y que pasamos a describir, estableciendo las diferencias entre los subgrupos. La edad media de Dependencia (D) es mayor que la de Consumo Perjudicial (CP) y de Intoxicación (I). (Ver Tabla 2). Las diferencias por sexo muestran un predominio del sexo masculino: 171 varones (91\%) por 17 mujeres (9\%). Respecto a la forma de convivencia resalta la mayor proporción de $\mathrm{D}$ que vive con familia propia $(47 \%)$ respecto a CP (26\%), al revés que con la familia de origen ( $D=22.7 \%$ y $C P=43,2 \%$ ) y sobre todo en el apartado otros que indica formas de convivencia en el desarraigo $(D=7,6 \%, C P=21,2 \%)$. (Ver Tabla 3).

Tabla 2. Distribución de la edad por diagnóstico.

\begin{tabular}{||l|l|l||}
\hline Edad & Rango & Edad Media \\
\hline Dependencia & De 27 a 68 años & 40,3 años \\
\hline Perjudicial & De 19 a 80 años & 34,6 años \\
\hline Intoxicación & De 18 a 35 años & 27,0 años \\
\hline
\end{tabular}


Tabla 3. Distribución del tipo de convivencia en función de la variable tipo de consumo

\begin{tabular}{|l|l|l|l|l||}
\hline & Vive Solo & Familia & Familia de & Otros \\
& & propia & origen & \\
\hline Dependencia & 18 & 37 & 18 & 6 \\
Perjudicial & 10 & $(47 \%)$ & $(22.7 \%)$ & $(7.6 \%)$ \\
\hline Intoxicación & $(9.6 \%)$ & $(26 \%)$ & $(43.2 \%)$ & $(21.2 \%)$ \\
\hline Total & $(40 \%)$ & $(20 \%)$ & $(40 . \%)$ & $(0.0 \%)$ \\
\hline \hline
\end{tabular}

Tabla 4. Nivel de estudios en función de la variable tipo consumo.

\begin{tabular}{|c|c|c|c|c|}
\hline & Sin Estudios & Primarios & \begin{tabular}{|l|} 
Secundarios \\
F.P. O Bachiller
\end{tabular} & Universitarios \\
\hline Dependencia & $\begin{array}{l}15 \\
(19 \%)\end{array}$ & $\begin{array}{l}38 \\
(48,1 \%)\end{array}$ & $\begin{array}{l}19 \\
(24 \%)\end{array}$ & $\begin{array}{l}7 \\
(8,9 \%)\end{array}$ \\
\hline $\begin{array}{l}\text { Consumo } \\
\text { Perjudicial }\end{array}$ & $\begin{array}{l}4 \\
(3,8 \%)\end{array}$ & $\begin{array}{l}66 \\
(63,5)\end{array}$ & $\begin{array}{l}27 \\
(26 \%)\end{array}$ & $\begin{array}{l}7 \\
(6.7 \%)\end{array}$ \\
\hline Intoxicación & 0 & $\begin{array}{l}5 \\
(100 \%)\end{array}$ & 0 & 0 \\
\hline Total & $\begin{array}{l}19 \\
(10,1 \%)\end{array}$ & $\begin{array}{l}109 \\
(57,9 \%)\end{array}$ & $\begin{array}{l}46 \\
(24,5 \%)\end{array}$ & $\begin{array}{l}14 \\
(7,5 \%)\end{array}$ \\
\hline
\end{tabular}

Tabla 5. Actividad laboral en función de la variable tipo de consumo.

\begin{tabular}{|c|c|c|c|c|}
\hline & Paro & Activo & Invalidez & Jubilado \\
\hline Dependencia & $\begin{array}{l}34 \\
(43 \%)\end{array}$ & $\begin{array}{l}28 \\
(35,4 \%)\end{array}$ & $\begin{array}{l}12 \\
(15,2 \%)\end{array}$ & $\begin{array}{l}5 \\
(6,4 \%)\end{array}$ \\
\hline $\begin{array}{l}\text { Consumo } \\
\text { Perjudicial }\end{array}$ & $\begin{array}{l}66 \\
(63,4 \%)\end{array}$ & $\begin{array}{l}33 \\
(31,7 \%)\end{array}$ & $\begin{array}{l}3 \\
(2,9 \%)\end{array}$ & $\begin{array}{l}2 \\
(2 \%)+\end{array}$ \\
\hline Intoxicación & $\begin{array}{l}2 \\
(40 \%)\end{array}$ & $\begin{array}{l}3 \\
(60 \%)\end{array}$ & 0 & 0 \\
\hline Total & $\begin{array}{l}102 \\
(54,2 \%)\end{array}$ & $\begin{array}{l}64 \\
(34 \%)\end{array}$ & $\begin{array}{l}15 \\
(8 \%)\end{array}$ & $\begin{array}{l}7 \\
(3,8 \%)\end{array}$ \\
\hline
\end{tabular}

El análisis del nivel de estudios, podría indicar una pequeña decantación del grupo $D$ hacia menor nivel de estudios, comparado con el grupo CP. (Ver Tabla 4).

El nivel de actividad laboral diferencia mas claramente entre $\mathrm{D}$ y CP sobre todo en la proporción de población en paro y en situación de invalidez y jubilación. (Ver Tabla 5). El grupo D es algo mayor en edad, pero también explica la diferencia la mayor proporción de enfermedades como vamos a ver a continuación.

Tabla 6. Enfermedades atribuidas al consumo de alcohol, según diagnóstico.

\begin{tabular}{||l|l|l|l||}
\hline \multicolumn{1}{|c|}{ Enfermedad } & Dependencia & Consumo & Intoxicación \\
\hline Encefalopatía alcohólica & 3 & 0 & 0 \\
\hline Neuropatía & 1 & 0 & 0 \\
\hline Esofagitis & 2 & 0 & 0 \\
\hline Gastritis & 0 & 8 & 0 \\
\hline Ulcera gastroduodenal & 4 & 1 & 0 \\
\hline Hepatopatía. Esteatosis & 11 & 2 & 0 \\
\hline Hepatitis & 18 & 5 & 0 \\
\hline Cirrosis & 5 & 1 & 0 \\
\hline Pancreatitis & 2 & 0 & 0 \\
\hline Miocardiopatia alcohólica & 3 & 0 & 0 \\
\hline Hipertensión arterial & 5 & 1 & 0 \\
\hline Traumatismos. Fracturas & 4 & 0 & 0 \\
\hline Anemia & 1 & 0 & 0 \\
\hline Hiperuricemia & 3 & & \\
\hline \hline
\end{tabular}

En lo que se refiere a la tabla 6 , es lógico suponer que las personas que tengan un consumo continuado de alcohol pueden tener mayores complicaciones a causa de dicha ingesta. Por lo tanto no sorprende el mayor número de enfermedades encontradas en los pacientes con un patrón de consumo continuado (D), tales como las referidas al sistema digestivo. De igual modo ocurre al considerar los trastornos orgánicos cerebrales secundarios al consumo de alcohol. (Ver Tabla 7). 
Tabla 7. Trastornos orgánicos cerebrales según diagnósticos.

\begin{tabular}{||l|l|l|l||}
\hline Trastorno orgánico cerebral & Dependencia & Consumo Perjudicial & Intoxicación \\
\hline 1- Secundario a otro trastorno & & & \\
\hline a)Demencia Sida & 1 & 0 & 0 \\
\hline b)Trauma Craneoencefalico & 1 & 0 & 0 \\
\hline 2 Alcohólico: & & & \\
\hline a)Delirium & & & \\
\hline b)Trast. Orgánico personalidad & 4 & 0 & 0 \\
\hline c)Trastorno cognitivo & 6 & 2 & 0 \\
\hline d)Demencia & 2 & 1 & 0 \\
\hline e)Trastorno delirante & 1 & 1 & 0 \\
\hline f)Alucinosis & 0 & 0 & 0 \\
\hline g)Amnesia & 2 & & 0 \\
\hline
\end{tabular}

En los trastornos psiquiátricos, como Esquizofrenia o Trastorno bipolar, se observa una mayor proporción de dichos diagnósticos en el grupo CP, lo que lleva a pensar en una mayor probabilidad de conductas de riesgo como sería el consumo perjudicial de alcohol en los enfermos psicóticos que por su enfermedad previa no llegan a desarrollar dependencia en sentido estricto. Coinciden nuestros datos con lo indicado otros investigadores(24). La Fase Maníaca puede acompañarse de consumo desproporcionado de alcohol, de acuerdo con la elevación del estado ánimo, con criterios de $\mathrm{CP}$, pero el consumo cedería al remitir la fase.(Ver Tabla 8 )

Tabla 8. Trastornos psicóticos en función de la variable tipo de consumo

\begin{tabular}{|l|l|l|l||}
\hline \hline & Dependencia & Consumo & Intoxicación \\
Esquizofrenia $(\mathrm{n}=40)$ & $4(57 \%)$ & $14(37 \%)$ & 0 \\
a)Paranoide & $3(43 \%)$ & $2(5,2 \%)$ & 0 \\
b)Esquizoafectiva & 0 & $17(44,7 \%)$ & 0 \\
c)Indiferenciada & 0 & $5(13,1 \%)$ & 0 \\
\hline Trastorno Bipolar $(\mathrm{n}=5)$ & 0 & & \\
\hline \hline
\end{tabular}

Respecto a los trastornos de ansiedad, se puede notar su práctica ausencia en nuestra muestra en contra de lo descrito por autores como Sellman (25). (Ver Tabla 9). Por otro lado la mayor proporción de trastornos afectivos en el grupo D, corrobora lo citado por diversos autores $(26,27)$ e indica también una diferencia entre $C$ y $C P$.

\section{Tabla 9. Trastornos del humor y de ansiedad en función de la variable tipo de consumo.}

\begin{tabular}{|c|c|c|c|}
\hline & Dependencia & Consumo & Intoxicación \\
\hline Trastornos del Humor (Afectivos) & & & \\
\hline a)Episodio depr.leve-moderado & $8(27,5 \%)$ & $3(15,6 \%)$ & 0 \\
\hline b)Episodio depres. Grave & $4(13,8 \%)$ & $4(21,1 \%)$ & 0 \\
\hline c)Trast. Depres, Recurrente & $1(3,4 \%)$ & 0 & 0 \\
\hline d)Distimia & $5(17,5 \%)$ & 0 & 0 \\
\hline Trastornos de ansiedad y fóbicos & & & \\
\hline a)Trast. Ansiedad generalizada & $1(3,4 \%)$ & 0 & 0 \\
\hline Trastornos a estres y adaptativos & $10(34,4 \%)$ & $12(60,3 \%)$ & $2(100 \%)$ \\
\hline
\end{tabular}

En el resto de trastornos psiquiátricos estudiados se observa una clara diferencia en los diagnósticos de Abuso de Sustancias no alcohólicas, Trastornos de Personalidad Trastornos del Control de Impulsos (ludopatía) y la conducta suicida asociada encontrándose mayor numero de casos en el grupo CP que en el D (Ver Tabla10), coincidiendo con otros autores $(28,29$, 30, 31).

Tabla 10. Otros trastornos psiquiátricos no citados previamente en función a la variable tipo de consumo

\begin{tabular}{||l|l|l|l||}
\hline & Dependencia & Consumo & Intoxicación \\
\hline Trast. Somatomorfos & 1 & 0 & 0 \\
\hline Trast. Conducta Alimentaria & 0 & 2 & 0 \\
\hline Trast. Identidad Sexual & 0 & 1 & 0 \\
\hline Trast. Por Abuso de Substancias & 24 & 81 & 0 \\
\hline Trast. Personalidad & 11 & 26 & 1 \\
\hline Trast. Impulsos(ludopatía) & 0 & 5 & 0 \\
\hline \hline
\end{tabular}


En la Tabla 11 se muestra el número de ingresos en función del tipo de consumo, incluyendo el número de ingresos previos tomados de los antecedentes personales de los pacientes. Así se refleja una mayor presión asistencial en el grupo CP.

\section{Tabla 11. Indicadores asistenciales en función de la variable tipo de consumo.}

\begin{tabular}{||l|l|l|l||}
\hline \hline & Dependencia & Consumo & Intoxicación \\
\hline $\begin{array}{l}N^{0} \text { Ingresos Psiquiátricos } \\
\text { (incluyendo ingresos previos) }\end{array}$ & 78 & 145 & 7 \\
\hline $\begin{array}{l}\text { Días de Estancia : } \\
\text { a) Rango } \\
\text { b) Media }\end{array}$ & 1 a 58 días & 1 a 83 días & 1 a 3 días \\
11,8 días & 12.6 días & 2 días \\
\hline
\end{tabular}

\section{DISCUSION.}

Los estudios epidemiológicos como el ECA (Epidemiological Catchmente Area) descrito por Regier (32), indican la preocupación por el impacto sanitario secundario al alcoholismo y la creciente presencia de trastornos psiquiátricos asociados. En la tabla 12 ofrecemos una visión sintetizada de una revisión de la bibliografía de los últimos diez años, que indica como la comorbilidad psiquiátrica en el alcoholismo, preocupa en naciones de nuestra área de influencia, como U.S.A. ( en diferentes estratos y grupos sociales), Alemania, U.K., o Australia. Nuestro trabajo señala la dificultad de extrapolar los resultados pertenecientes a trabajos realizados en distintas poblaciones. Resaltamos que nuestros resultados se basan en los ingresos de una unidad de psiquiatría, situada en un hospital general.

Tanto los estudios clínicos citados, como nuestros resultados, confirman la existencia de una importante patología psiquiátrica asociada, al diagnóstico de alcoholismo. Pero hay una gran variación en las poblaciones estudiadas, unas provienen de clínicas de deshabituación, otros se refieren a estudios epidemiológicos a escala nacional y otros estudian los pacientes ingresados en hospital general. No se trata de poblaciones homogéneas, ya que el lugar donde se haya diagnosticado la enfermedad alcohólica puede sesgar el conjunto de datos recogidos, siendo un caso evidente los Hospitales de Veteranos de los USA.

El examen de los métodos diagnósticos indica que existen diferencias de unos trabajos a otros. El estudio de Tomasson (6) a escala Nacional en Islandia
Tabla 12. Estudios de comorbilidad

\begin{tabular}{|c|c|c|}
\hline AUTOR Y PAÍS & $\begin{array}{l}\text { POBLACIÓN } \\
\text { PATOLOGÍA }\end{array}$ & SETTING \\
\hline Vuksic/Croacia(33) & $\begin{array}{l}\text { Veteranos } \\
\text { PTSD-ansiedad }\end{array}$ & Consultas Externas \\
\hline Pettinati/USA(29) & $\begin{array}{l}\text { Toxicómanos } \\
\text { Tr.personalidad }\end{array}$ & Clínica.Deshabituación \\
\hline Sullivan/UK(27) & $\begin{array}{l}\text { Nacional } \\
\text { Depresión }\end{array}$ & Estudio Nacional \\
\hline Federman/USA(22) & $\begin{array}{l}\text { Indios Adolescentes. } \\
\text { Consumo substancias }\end{array}$ & Estudio Rural \\
\hline Lambert/USA(34) & $\begin{array}{l}\text { Enfermos Psiq. } \\
\text { Abuso substancias. }\end{array}$ & Hosp.Psiquiátrico. \\
\hline Fils-Aime/Suec(35) & Alcoholicos remitidos & Hosp. Investigación \\
\hline Schuckit/USA(36) & Hijos de alcohólicos. & Estudio local. \\
\hline Tomasson/Islan(6) & Alcohólicos & Clínica Deshabituación. \\
\hline Haver/Suecia(37) & Mujeres de alcohólicos & Clínica Deshabituación \\
\hline
\end{tabular}

utiliza el criterio de la DSM-III, y unifica el diagnóstico de Dependencia y Abuso. Arolt y Driessen (2) estudian la población ingresada en un hospital general, utilizando el DSM: III-R y hacen la diferencia entre:

- ocurrencia de alcoholismo a lo largo de la vida y

- alcoholismo presente

pero sin especificar la forma de consumo alcohólico. Si en nuestra muestra no hubiéramos incluido tres grupos sub-diagnósticos de alcoholismo, habríamos encontrado un espectro de enfermedades psiquiátricas parecido al de estos autores. Pero al diferenciar el alcoholismo en tres grupos creemos haber encontrado diferencias entre la dependencia y el consumo, como se refleja en el análisis de las tablas de los 
resultados. En nuestro trabajo falta un estudio estadístico y una mayor precisión en la valoración en las variables de presión asistencial. Al encontrar en las tablas valores inferiores a cinco, no hemos podido utilizar el contraste chi2.

Las conclusiones del presente trabajo indican la existencia de tres patrones diferenciados de consumo de sustancias alcohólicas en los enfermos ingresados a lo largo de tres años en un servicio de psiquiatría de un Hospital general:

Intoxicación. Es un consumo esporádico característico de personas jóvenes, que no requiere ingreso mas que ocasionalmente, la mayor parte de los casos no pasa de las salas de urgencias. No hay patología somática y el diagnóstico psiquiátrico mas habitual es el de Trastorno Adaptativo.

Consumo Perjudicial. Se trata de un grupo de personas con una media de edad de 34 años, con tendencia a la marginalidad y el desarraigo. No es frecuente la patología somática secundaria al consumo de alcohol excepto la producida por ingesta aguda como podría ser gastritis. Tampoco hay Trastornos Orgánicos Cerebrales secundarios al alcohol. Los diagnósticos psiquiátricos mas frecuentes son:

- Trastornos por el uso, consumo y dependencia de sustancias

- Trastornos de personalidad

- Trastornos por control de impulsos

- Psicosis (Esquizofrenia y PMD).

Dependencia. Aquí no encontramos con lo que siempre se ha entendido como alcoholismo. Es un grupo de edad de 40 años, con familia propia, o que vive solo, y con problemas de jubilación e invalidez. Presenta la habitual patología orgánica asociada al consumo prolongado de alcohol, y Trastornos Orgánicos Cerebrales secundarios al alcoholismo. El diagnóstico psiquiátrico mas habitual es el de Trastornos del Humor o de la Afectividad.

\section{CONCLUSIONES.}

Se evidencian tres tipos de consumo alcohólico bastante diferenciados:

El grupo de Intoxicación con personas jóvenes con trastornos emocionales pasajeros que no tienen repercusiones somáticas ni psiquiátricas.

El grupo de alto consumo de riesgo, Consumo Perjudicial o Abuso, en el que se incluyen tanto las bebidas alcohólicas como cualquier otra sustancia de consumo, en el que predomina una gran inestabilidad social, familiar y alteraciones psiquiátricas graves.
Y por último tendríamos la Dependencia Alcohólica: el grupo de edad madura con mayor secuela de problemas orgánicos y cerebrales y tendencia al aislamiento y la depresión.

No puede afirmarse categóricamente que nos encontremos con tres grupos de enfermos separados herméticamente. Hay un solapamiento entre los tres. Además muchos individuos pueden pasar a lo largo del tiempo, de una a otra categoría. Según las conclusiones de este trabajo, la correcta identificación de estos grupos permitiría una mejor valoración de su impacto sociosanitario y tambien de su adecuado tratamiento.

\section{BIBLIOGRAFIA .}

1 - DSM-IV. Masson S.A. 1995

2 - Arolt-V; Driessen-M. Alcoholism and psychiatric comorbidity in general hospital inpatients. Gen-Hosp-Psychiatry. 1996 Jul; 18(4): 271-7.

3 - Hovens-JG; Cantwell-DP; Kiriakos-R. Psychiatric comorbidity in hospitalized adolescent substance abusers. JAm-Acad-Child-Adolesc-Psychiatry. 1994 May; 33(4): 476-83.

4 - Ross-HE. DSM-III alcohol abuse and dependence and psychiatric comorbidity in Ontario: results from the Mental Health Supplement to the Ontario Health Survey. Drug-Alcohol-Depend. 1995 Aug; 39(2): 111-28.

5 - Valbuena,A. y cols. "Peso asistencial de las complicaciones del alcoholismo". Congreso de la Sociedad Española de Psiquiatría Biológica. COMUNICACIÓN. Sevilla. 1995.

6 - Tomasson-K; Vaglum-P. A nationwide representative sample of treatment-seeking alcoholics: a study of psychiatric comorbidity. Acta-Psychiatr-Scand. 1995 Nov; 92(5): 378-85.

7 - Johnson-JG; Spitzer-RL; Williams-JB; Kroenke-K; LinzerM; Brody-D; deGruy-F; Hahn-S. Psychiatric comorbidity, health status, and functional impairment associated with alcohol abuse and dependence in primary care patients: findings of the PRIME MD-1000 study. J-Consult-Clin-Psychol. 1995 Feb; 63(1): 133-40.

8 - Cuadrado-Callejo-P. Alcohol dependence and psychiatric comorbidity. II. Clinical and therapeutic implications. Actas-Luso-Esp-Neurol-Psiquiatr-Cienc-Afines. 1997 Jan-Feb; 25(1): 34-44.

9 - Dawes-MA; Frank-S; Rost-K. Clinician assessment of psychiatric comorbidity and alcoholism severity in adult alcoholic inpatients. Am-J-Drug-Alcohol-Abuse. 1993; 19(3): 377-86.

10 - Miller-NS. Comorbidity of psychiatric and alcohol/drug disorders: interactions and independent status. JAddict-Dis. 1993; 12(3): 5-16.

11 - Cuadrado-Callejo-P. Alcohol dependence and psychiatric comorbidity. I. Epidemiology and etiopathogenic 
involvement. Actas-Luso-Esp-Neurol-Psiquiatr-CiencAfines. 1996 Sep-Oct; 24(5): 231-44.

12 - Pozzi-G; Bacigalupi-M; Tempesta-E. Comorbidity of drug dependence and other mental disorders: a two-phase study of prevalence at outpatient treatment centres in Italy. Drug-Alcohol-Depend. 1997 Jun 6; 46(1-2): 69-77

13 - Bleich-A; Koslowsky-M; Dolev-A; Lerer-B. Post-traumatic stress disorder and depression. An analysis of comorbidity. Br-J-Psychiatry. 1997 May; 170: 479-82.

14 - Katz-IR; Streim-J; Parmelee-P. Prevention of depression, recurrences, and complications in late life. Prev-Med. 1994 Sep; 23(5): 743-50.

15 - Ross-R; Booth-BM; Russell-DW; Laughlin-PR; Brown-K. Outcome of domiciliary care after inpatient alcoholism treatment in male veterans. J-Subst-Abuse-Treat. 1995 Sep-Oct; 12(5): 319-26.

16 - El-Guebaly-N. Alcohol and polysubstance abuse among women. Can-J-Psychiatry. 1995 Mar; 40(2): 73-9.

17 - Hill-SY. Vulnerability to alcoholism in women. Genetic and cultural factors. Recent-Dev-Alcohol. 1995; 12: 928.

18 - Hill-SY. Mental and physical health consequences of alcohol use in women. Recent-Dev-Alcohol. 1995; 12: 181-97.

19 - Smith-EM; North-CS; Spitznagel-EL. Alcohol, drugs, and psychiatric comorbidity among homeless women: an epidemiologic study. J-Clin-psychiatry. 1993 Mar; 54(3): 82-7.

20 - Westermeyer-J; Specker-S; Neider-J; Lingenfelter-MA. Substance abuse and associated psychiatric disorder among 100 adolescents. J-Addict-Dis. 1994; 13(1): 6789.

21 - Rohde-P; Lewinsohn-PM; Seeley-JR. Psychiatric comorbidity with problematic alcohol use in high school students. J-Am-Acad-Child-Adolesc-Psychiatry. 1996 Jan; 35(1): 101-9.

22 - Federman-EB; Costello-EJ; Angold-A; Farmer-EM; Erkanli-A. Development of substance use and psychiatric comorbidity in a epidemiologic study of white and American Indian young adolescents the Great Smoky Mountains Study. Drug-Alcohol-Depend. 1997 Mar 14; 44(2-3): 69-78.

23 - Liberto-JG; Oslin-DW; Ruskin-PE. Alcoholism in older persons: a review of the literature. Hosp-CommunityPsychiatry. 1992 Oct; 43(10): 975-84.

24 - Strakowski-SM; Keck-PE Jr; McElroy-SL; LonczackHS; West-SA. Chronology of comorbid and principal syndromes in first-episode psychosis. ComprPsychiatry. 1995 Mar-Apr; 36(2): 106-12.
25 - Sellman-JD; Eggleston-JE. Characteristics of people admitted for residential alcohol and drug treatment. NZ-Med-J. 1991 Jun 12; 104(913): 230-2.

26 - Sherbourne-CD; Wells-KB; Hays-RD; Rogers-W; Burnam-MA; Judd-LL. Subthreshold depression and depressive disorder: clinical characteristics of general medical and mental health speciality outpatients.

27 - Sullivan-PF; Kessler-RC; Kendler-KS. Latent class analysis of lifetime depressive symptoms in the national comorbidity survey. Am-J-Psychiatry. 1998 Oct; 155(10): 1398-406.

28- Diwiddie-SH; Reich-T; Cloninger-CR. Psychiatric comorbidity and suicidality among intravenous drug users. JClin-Psychiatry. 1992 Oct; 53(10): 364-9.

29- Pettinati-HM; Pierce-JD Jr; Belden-PP; Meyers-K. The relationship of Axis II personality disorders to other known predictors of addiction treatment outcome. AmJ-Addict. 1999 Spring; 8(2): 136-47

30- Allgulander-C; Allebeck-P; Przybeck-TR; Rice-JP. Risk of suicide by psychiatric diagnosis in Stockholm County. Eur-Arch-Psychiatry-Clin-Neurosci. 1992; 241(5): 323-6.

31- Michalon-M. Consultation-liaison psychiatry a prospective study in a general hospital milieu. Can-J-Psychiatry. 1993 Apr; 38(3): 168-74.

32- Regier, D.A., Boyd JH, Burje JD et als. One month prevalence of mental disorders. Arch. Gen. Psychiatry. 1988;45:977-986.

33 - Vuksic-Mihaljevic-Z; Mandic-N; Mihaljevic-S; IvandicA. Symptom structure and psychiatric comorbidity of combat related post-traumatic stress disorder. Psychiatry-Clin-Neurosci. 1999 Jun; 53(3): 343-9

34 - Lambert-MT; Griffith-JM; Hendrickse-W. Characteristics of patients with substance abuse diagnoses on a general psychiatry unit in a VA Medical Center. Psychiatr-Serv. 1996 Oct; 47(10): 1104-7

35 - Fils-Aime-ML; Eckardt-MJ; George-DT; Brown-GL; Mefford-I; Linnoila-M. Early-onset alcoholics have lower cerebrospinal fluid 5-hidroxyindoleacetic acid levels than late-onset alcoholics. Arch-Gen-Psychiatry. 1996 Mar; 53(3): 211-6

36 - Schuckit, M.A. Smith, T.L. An 8-year follow-up of 450 sons of alcoholic and control subjets. Arch. Gen. Psychiat. 1996/53:202-10.

37 - Haver-B; Dahlgren-L. Early treatment of women alcohol addiction (EWA): a comprehensive evaluation and outcome study. I. Patterns of psychiatric comorbidity at intake. Addiction. 1995 Jan; 90(1): 101-9 